\title{
Long Non-coding RNA Based Cancer Classification using Deep Neural Networks
}

\author{
Abdullah Al Mamun and Ananda Mohan Mondal \\ \{mmamu009,amondal\}@fiu.edu \\ Florida International University, Miami, FL
}

\begin{abstract}
Recent studies indicate that lncRNA plays key roles in tumorigenesis and misexpression of lncRNAs can lead to change in expression profiles of various target genes involved in different aspects of cancer progression. However, research on classifying multiple cancer types using only lncRNA is rarely found. In this paper, we explored the capability of lncRNA in classifying cancer types by employing four deep neural networks - multi-layer perceptron (MLP), longshort-term memory (LSTM), convolutional neural network (CNN) and deep autoencoder (DAE). For experiment, RNA-seq expression values from TCGA for 8 cancers - BLCA, CESC, COAD, HNSC, KIRP, LGG, LIHC, and LUAD - are used. The combined dataset consists of 3656 patients with expression values for 12309 lncRNAs. The performance of the models in terms of accuracy ranges from $94 \%$ to $98 \%$, which shows lncRNA expression profiles as the better signature compared to the mRNA expression profiles in classifying cancer types.
\end{abstract}

\section{KEYWORDS}

Cancer classification; lncRNA; RNA-seq; GDC-TCGA; CNN; DAE; LSTM; MLP

\section{ACM Reference Format:}

Abdullah Al Mamun and Ananda Mohan Mondal. 2019. Long Non-coding RNA Based Cancer Classification using Deep Neural Networks. In 10th ACM International Conference on Bioinformatics, Computational Biology and Health Informatics (ACM-BCB '19), September 7-10, 2019, Niagara Falls, NY, USA. ACM, New York, NY, USA, 1 page. https://doi.org/10.1145/3307339.3343249

Mis-expression of lncRNAs can lead to change in expression profiles of various target genes related to cell homeostasis [1]. Cheetham et. al. identified different $\ln \mathrm{cRNAs}$ th at are actively in volved in different stages of cancer development [1].

Few studies developed models for classification of multiple cancer types. For example, Li et. al. developed GA/KNN to classify cancer and normal samples for 31 cancers using RNA-seq gene expression data [2].

In this paper, we aim to develop models for classifying multiple cancer types using lncRNA expression employing deep neural networks.

Permission to make digital or hard copies of part or all of this work for personal or classroom use is granted without fee provided that copies are not made or distributed for profit or commercial advantage and that copies bear this notice and the full citation on the first page. Copyrights for third-party components of this work must be honored. For all other uses, contact the Owner/Author.

ACM-BCB '19, September 7-10, 2019, Niagara Falls, NY, USA

(C) 2019 Copyright is held by the owner/author(s).

ACM ISBN 978-1-4503-6666-3/19/09.

https://doi.org/10.1145/3307339.3343249
To validate the idea, we downloaded (April, 2019) RNA-seq FPKM normalized expression data for 8 cancers from UCSC xena [3].

The dataset has 12309 common lncRNA IDs with expression data for all selected cancers. The number of combined list of samples is 3656 after merging all the cancers.

For training $75 \%$ of each cancer type is selected randomly using seed 123 for random number generation. The remaining $25 \%$ is used for testing. This training and testing procedure has been repeated 10 times. The average of this 10 results are used as the performance of the model. Python is used for pre-processing and deeplearning4J, a java machine learning package is used for model development. All models are executed on a CPU Intel core i7 with 16GB RAM.

Four different performance metrics - accuracy, precision, recall, and F1 score - are measured to compare the model performance.

Table 1 shows the values of performance metrics for MLP, LSTM, CNN and DAE models. It is clear from the table that MLP has the

Table 1: Performance Comparison

\begin{tabular}{ccccl}
\hline Model Name & Accuracy & Precision & Recall & F1 \\
\hline MLP & 0.9371 & 0.9324 & 0.9290 & 0.9394 \\
LSTM & 0.9562 & 0.9508 & 0.9521 & 0.9514 \\
CNN & $\mathbf{0 . 9 7 8 1}$ & $\mathbf{0 . 9 7 6 5}$ & $\mathbf{0 . 9 7 6 4}$ & $\mathbf{0 . 9 7 6 4}$ \\
DAE & 0.9639 & 0.9613 & 0.9590 & 0.9600 \\
\hline
\end{tabular}

lowest accuracy of $94 \%$ and CNN has the highest accuracy of $98 \%$. Similarly, MLP has the lowest precision, recall, and F1 scores nearly 93\% while CNN has the highest score, $98 \%$, for all metrics.

Present study shows that lncRNA expression is a significant feature to differentiate multiple cancer types, which is evidenced by the performance of the proposed models that are able to correctly classify nearly $98 \%$ of the cancer samples.

\section{ACKNOWLEDGMENT}

This research is funded by NSF CAREER award \#1651917 (transferred to \#1901628) to AMM.

\section{REFERENCES}

[1] SW Cheetham, F Gruhl, JS Mattick, and ME Dinger. Long noncoding rnas and the genetics of cancer. British journal of cancer, 108(12):2419, 2013.

[2] Yuanyuan Li, Kai Kang, Juno M Krahn, Nicole Croutwater, Kevin Lee, David M Umbach, and Leping Li. A comprehensive genomic pan-cancer classification using the cancer genome atlas gene expression data. BMC genomics, 18(1):508, 2017.

[3] Mary Goldman, Brian Craft, Mim Hastie, Kristupas Repečka, Akhil Kamath, Fran McDade, Dave Rogers, Angela N Brooks, Jingchun Zhu, and David Haussler. The ucsc xena platform for cancer genomics data visualization and interpretation. BioRxiv, page 326470, 2019. 